\title{
Optimal Trajectories Computation Within Regions of Interest for Hepatic RFA Planning
}

\author{
Caroline Villard ${ }^{1}$, Claire Baegert ${ }^{1}$, Pascal Schreck ${ }^{1}$, \\ Luc Soler $^{2}$, and Afshin Gangi ${ }^{3}$ \\ 1 LSIIT, Pôle API, F67400 Illkirch, France \\ \{villard, baegert, schreck\}@lsiit.u-strasbg.fr \\ 2 IRCAD, 1 place de l'Hôpital, F67091 Strasbourg, France \\ 3 Hôpital Civil, Radiologie B, 1 place de l'Hôpital, F67091 Strasbourg, France
}

\begin{abstract}
Percutaneous radiofrequency ablation has become a frequently used technique for the treatment of liver cancers, but still remains very difficult to plan. In this paper, we propose a robust method to delineate on the skin of a $3 \mathrm{D}$ reconstructed patient the zones that are candidate for an insertion, because they allow a safe access to the tumor without meeting any organ, and to compute automatically within these zones an optimal trajectory minimizing the volume of necrosis covering the tumor.
\end{abstract}

\section{Introduction}

Radiofrequency ablation (RFA) of liver tumors is a relatively recent technique, that has been increasingly used in the past few years. The percutaneous procedure has proved its effectiveness, relative safety and predictability. It has the advantage to be minimally invasive, that means lighter operations and shorter hospital stays, becoming a good option for unresectable cases or small tumors.

This approach consists in inserting a probe through the skin towards the tumor, and causing coagulative necrosis of the tumor by heating the tissues surrounding the probe's tip above $60^{\circ} \mathrm{C}$ thanks to an ionic agitation due to the principle of microwave. The success of such an operation closely depends on the choice of an optimal strategy for the insertion of the RF probe through the skin, even though this choice remains very difficult for a physician, who can only rely on 2D slices acquired from CT scan or MRI.

Our long term objective is to elaborate a complete tool for patient-specific treatment planning, surgeons training, and even robotically assisted interventions, including all steps from the $3 \mathrm{D}$ reconstruction of the acquired images to simulation and assistance, including image analysis, 3D modeling, 3D interaction, haptics and virtual reality, augmented reality, automatic planification, and robotics. In this paper, we will only focus on one part of this work, currently in progress, concerning the automatic planification of an appropriate strategy for needle placement, and detail our first encouraging results.

After a brief state of the art in Section 2, we will expose our new method in Sections 3 and 4 . Then we will discuss the results, report the few remaining problems of our approach, and give perspectives for our future works. 


\section{Previous Works}

\subsection{General}

First of all, our work is based on an abundant literature about RFA, explaining widely the profile of candidate patients, the principle and the effects of the process, existing devices and usual treatment strategies, the nature of possible complications, the reasons why complications or failure may occur and the occurring rates, and the procedures that improve the treatment, for hepatic or other tumors, and for percutaneous, open, or laparoscopic procedures [1234].

In addition to a need from radiologists, our project also takes its motivations from works proving how an important part of the success of an intervention was played by the training and experience of the surgeons [5. That is why a realistic training simulator can be very useful in the formation of novices. Moreover, it has been underlined that an volumic view improved the success rates [6], so we think that helping the radiologists in having a better visualization, and even providing him an assistance for the treatment planning can be of valuable help.

In computer science, many works have been focused on simulations of cancer treatments, a few ones concerning RFA, or cryotherapy that has a lot of common points. Most of the developments use finite elements methods, reproducing the thermic exchanges within the teated area 7/8. However, this approach has the drawback to be quite slow, whereas one of our objectives is to have a low-cost and transportable solution, working on a common laptop. Treatment planning has been less studied for RFA, but there are close works in neurosurgery, where we find comparable objectives: destroying tumors and damaging as less surrouding tissue as possible, even if the constraints are not the same [9].

\subsection{Basis of Our Works}

The tool we are developping is based on works, presented a few years ago, about automatic 3D reconstruction of slices from enhanced spiral CT scans with $2 \mathrm{~mm}$ cuts acquired from patients with liver metastases [10]. The software detects, delineates and reconstructs automatically their liver, pathologies, and surrounding organs. It produces realistic and manipulable 3D scenes representing the anatomy of the patients.

Then, we added the possibility to perform simulations of RFA [11, based upon the characteristics of the Berchtold HITT needle. A user of the simulator can add virtual probes into the 3D scene of the patient's organs, and then freely manipulate them. During a simulation, the lesion zone is estimated and simulated as a simple mesh representing the $60{ }^{\circ} \mathrm{C}$ isosurface, most of the time approximated as being a simple spheroid, that is deformed when necessary to simulate the heat-sink effect caused by large vessels.

First attempts were also leaded to perform an automatic treatment planning for RFA [12. We proposed an algorithm able to find automatically a secure trajectory for the needle, covering the whole tumor plus an additional security margin while minimizing the damages on healthy cells and avoiding other organs. The first part of this algorithm finds the minimal spheroid containing the tumor 
given a fixed trajectory for the needle, and computes its volume. The second part is based on a classic minimization method, the Downhill Simplex algorithm [13, and tries to find the smallest minimal spheroid by varying a set of parameters corresponding to the needle's position and orientation. To avoid organs, we simply return a penalty volume for candidate trajectories that would meet an organ, in order to eliminate this candidate. With this method, we managed to obtain satisfying results in terms of volume, with acceptable computation times.

However, a few problems still remained. The major of them was that we observed that this approach was quite dependent on the initial position from which the process was launched. Two phenomena were involved. The first one was due to the Downhill Simplex method we use, that is known to be sensitive to local minima. The second one was due to the way we avoid organs: it leads the minimization process to be bounded into a zone delimited by the surrounding presence of organs. Then, if the initial position was located within such an area, the minimization process was not able to cross the virtual boundary, and was limited to the minimum of the considered area. Due to these problems, the planning could not be really considered as being fully automatic, as it depended on the initial data determined by the user. As we wanted it to be fully automatic, we tried to find ways to solve these problems. That is the main purpose of this paper, and we describe in next section the solutions we propose.

\section{Determination of the Candidate Zones for Needle Insertion}

The algorithm we propose acts in 3 phases. The first one is the delimitation of the zones of the skin where a needle can be inserted and reach the target without meeting an organ. Let us describe this phase more in detail.

\subsection{Simplified Algorithm}

If we consider the tumor as a visualization point, we have to solve a visibility problem. All the points that are visible from the tumor point of view without being hidden by any organ are candidates. Of course, the tumor cannot be considered as a single point of view, because it has a volume, but we'll explain later how we can extend the proposed algorithm. For simplicity and computing efficiency, we do not consider all the points of the surface of the skin, but only the triangles of the mesh. One triangle is considered as visible if every point in this triangle is visible, hidden otherwise. That means that if one triangle belongs to the accessibility zone, any needle insertion in this triangle that reaches the tumor (tumor considered as a point) will not collide any organ. Then we only have to determine the visibility of each skin's triangle from the tumor. To do this, we place a camera at the point of view, and compute 6 views of the scene, each one corresponding to one face of an imaginary projection cube around the tumor. If one triangle is hidden by an organ (except liver), there will be at least one view in which it will be detected. Triangles not detected as hidden are visible. 


\subsection{Algorithm Taking into Account Tumor's Volume}

Let's now consider the whole tumor volume. We will say that a triangle is visible from the tumor if it is visible from at least $p \%$ of its voxels. In practice, we will only consider triangles that have a $100 \%$ visibility, in order to ensure that the whole tumor is reachable from the zone without any obstructing organ. To compute the $100 \%$ visible triangles, we could launch the previous algorithm from each tumor's voxel, considering it as the projection cube. In order to optimize the algorithm, we only examine external voxels of the tumor. Among them, we only compute the views corresponding to faces adjacent to other external voxels. On Fig 1 the considered faces in the tumor are drawn in thick. For each view, if a triangle is hidden we eliminate it from the list of candidates, that will be called the $100 \%$ zone. An example of the $100 \%$ zone is shown on left of Fig.2.

The time taken to determine the $100 \%$ zone mainly depends on the number of tumor's external voxels, as seen in Table 1. but it is not the only influencing factor, as can be seen comparing cases 2 and 3, and cases 4 and 5, where the number of tumor's external voxels are quite the same but times are different. The complexity of the scene and the skin's mesh have a reduced but not insignificant influence on the execution time. We can see for instance in cases 1 and 2 that,
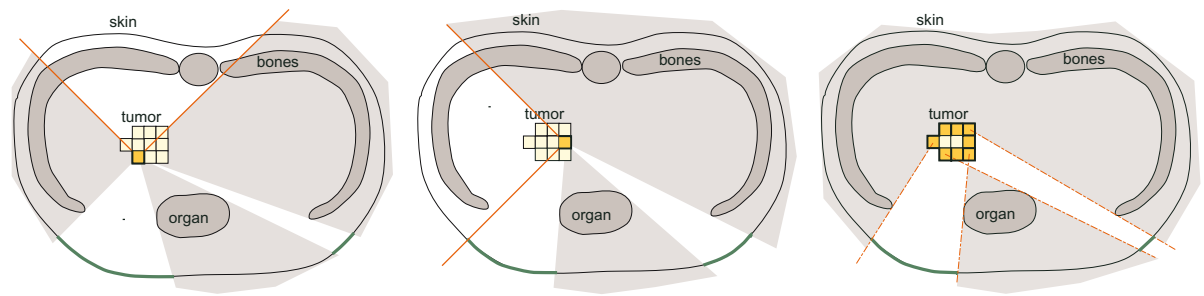

Fig. 1. Computation of the candidate zones on the skin
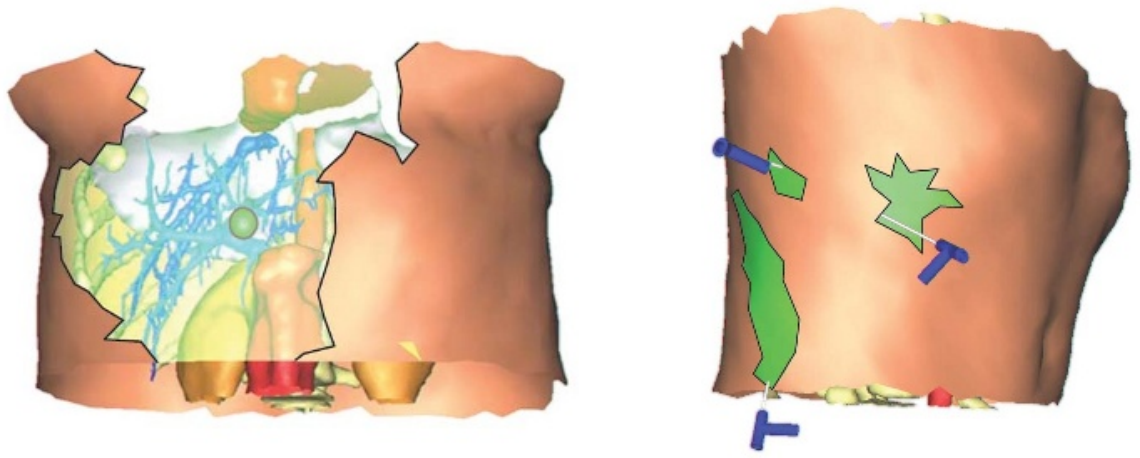

Fig. 2. Left: example of a computed $100 \%$ zone (in transparent with a thick border, 1 c.c.); Right: example of computed optimal trajectories for each of the 3 c.c. of the $100 \%$ zone (here in opaque with a thick border) 
Table 1. Execution times for the computation of candidate zones in 5 cases. The number and surfaces of the obtained zones can be found on Table 2

\begin{tabular}{|c|c|c|c|c|}
\hline case \# & $\begin{array}{c}\text { nb. of tumor's } \\
\text { external voxels }\end{array}$ & $\begin{array}{c}\text { nb. of skin's } \\
\text { triangles }\end{array}$ & $\begin{array}{c}\text { nb. of other } \\
\text { organs triangles }\end{array}$ & $\begin{array}{c}\text { execution } \\
\text { time (s) }\end{array}$ \\
\hline 1 & 220 & 2106 & 207257 & 43 \\
\hline 2 & 401 & 2055 & 150187 & 63 \\
\hline 3 & 417 & 2062 & 185509 & 74 \\
\hline 4 & 1198 & 2074 & 141285 & 186 \\
\hline 5 & 1120 & 1953 & 171401 & 175 \\
\hline
\end{tabular}

the execution time is barely the same, showing that a large number of triangles compensates for a small number of voxels. In cases 4 and 5, the number of skin's triangles seems to make the difference and to lengthen the process. Times are computed with a Pentium 4 with $1,5 \mathrm{GHz}$ and 768 Mo RAM and a Radeon 8500.

\section{Candidate Optimal Trajectory for Each Zone}

After the computation of each connected component, the goal is to launch the minimization algorithm in each component in order to compare the respective minima and choose the best one. In previous works, we used to choose randomly an initial position of the needle, and launch the minimization process. When a candidate trajectory collided an organ, the volume of the lesion was artificially increased. Here, for the second phase, we will use a quite similar approach.

On a first idea, we tried to launch the minimization from a randomly chosen triangle of the connected component. The results were satisfying for small, convex zones. But in larger zones, the algorithm often fell into local minima. We decided to add an initialization phase, to bring the initial position closer to the minimum. We make a quick estimation of the burnt volume for the barycentre of each candidate triangle, with the tip of the needle placed in the centre of the tumor's bounding box (not axis-aligned). Then we compare the obtained volumes and initialize the needle in the position of the smallest one. If the initial position corresponds to the good valley, the needle will reach the good minimum.

On Table 2 we can see the difference between the obtained minima, with or without initialization phase. In this table, we only mentioned connected components containing more than one triangle, because we consider zones with only one triangle as being too risky (too closely surrounded by organs). We can see on Fig 2 the result of this process for the three $100 \%$ zones obtained in case \#4.

We observe that the initialization phase is more or less efficient according to the size of the region. The bigger the region is, the more the minimization with initialization can improve the result: we notice that we obtain an average gain of $-0.213 \mathrm{~mL}$ for zones larger than $10 \mathrm{~cm}^{2}$, whereas we obtain no gain (or infinitesimal) for smaller zones. This is probably because in large regions there are more local minima in which the process could fall, and starting the process in the appropriate valley prevents more often from a wrong convergence. When the zone is small, the method provides approximately the same result in volume. 
Table 2. Results of the minimization for each candidate zone, for 5 patient cases, with and without initialization. Last column: objective to reach.

\begin{tabular}{|c|c|c|c|c|c|c|c|}
\hline \multirow{2}{*}{$\begin{array}{c}\text { case } \\
\#\end{array}$} & \multirow{2}{*}{$\begin{array}{c}\text { connected } \\
\text { component \# }\end{array}$} & \multirow{2}{*}{$\begin{array}{l}\text { size of the } \\
\text { component } \\
\left(\mathrm{cm}^{2}\right)\end{array}$} & \multicolumn{2}{|c|}{ without init. } & \multicolumn{2}{|c|}{ with init. } & \multirow{2}{*}{$\begin{array}{l}\text { theor. } \\
\text { min. vol } \\
(\mathrm{mL})\end{array}$} \\
\hline & & & $\begin{array}{l}\min . \text { vol. } \\
(\mathrm{mL})\end{array}$ & $\begin{array}{l}\text { time } \\
(\mathrm{s})\end{array}$ & $\begin{array}{l}\text { min. vol. } \\
(\mathrm{mL})\end{array}$ & $\begin{array}{c}\text { time } \\
(\mathrm{s})\end{array}$ & \\
\hline 1 & 1 & 234,4 & 3,588 & 12 & 3,067 & 13 & 2,730 \\
\hline \multirow[t]{6}{*}{2} & 1 & 1,1 & 7,227 & 18 & 7,222 & 16 & \multirow{6}{*}{6,830} \\
\hline & 2 & 1,6 & 7,180 & 12 & 7,180 & 12 & \\
\hline & 3 & 2,2 & 6,888 & 13 & 6,859 & 13 & \\
\hline & 4 & 2,3 & 7,532 & 8 & 7,534 & 8 & \\
\hline & 5 & 12,0 & 7,698 & 12 & 7,596 & 12 & \\
\hline & 6 & 92,0 & 7,070 & 15 & 7,073 & 13 & \\
\hline \multirow[t]{4}{*}{3} & 1 & 3,2 & 5,823 & 11 & 5,837 & 7 & \multirow[b]{4}{*}{3,059} \\
\hline & 2 & 5,0 & 5,846 & 10 & 5,827 & 8 & \\
\hline & 3 & 5,2 & 5,740 & 12 & 5,739 & 12 & \\
\hline & 4 & 150,6 & 3,858 & 26 & 3,831 & 14 & \\
\hline \multirow[t]{3}{*}{4} & 1 & 4,3 & 13,707 & 16 & 13,751 & 11 & \multirow[b]{3}{*}{8,876} \\
\hline & 2 & 10,8 & 12,270 & 15 & 11,960 & 13 & \\
\hline & 3 & 28,1 & 10,610 & 16 & 10,618 & 14 & \\
\hline \multirow[t]{3}{*}{5} & 1 & 12,0 & 13,698 & 20 & 13,678 & 13 & \multirow[b]{3}{*}{9,304} \\
\hline & 2 & $\overline{79,0}$ & 11,841 & 19 & 11,805 & 13 & \\
\hline & 3 & 162,9 & 10,216 & 18 & 9,301 & 20 & \\
\hline
\end{tabular}

Concerning execution time, we noticed that the minimization process itself converges faster when the needle is previously positioned. If we add initialization and minimization times the total time sometimes increases, but in most cases does not exceed the time witout initialization. We even have an average gain of -2.41 s. In conclusion, we think that the initialisation is always useful: for large regions it allows to provide a sizeable better volume, in other cases it speeds up the process.

On this table, we also mentioned in the last column the theoretical minimal covering volume that could be reached if the surrounding organs were not taken into account, that can be seen as a goal: for each case, this volume is written in front of the best candidate trajectory. This value is computed thanks to an exhaustive sampling method. Most of the time, this theoretical minimal volume doesn't correspond to a possible needle insertion point, but we can see that we manage to find a very close result within authorized areas, with an average of only $+0.57 \mathrm{~mL}$, i.e. $+11.52 \%$ of the theoretical values, that is encouraging.

\section{Discussion}

Until now, we always considered the optimal trajectory as being the one providing a minimal volume of burnt tissue, that was the aim of this work. However, we have to notice that a radiologist would not always make the same choice. 
First of all, we did not take into account some additional constraints, such as the length of the needle, or the level of risk. Sometimes, the trajectory is good, but impossible to reproduce in practice. To solve these problems, we plan to eliminate triangles being too far from the tumor, and to add an extra margin around organs, except bones that can usually be safely approached by the needle, in order to eliminate unfeasible insertion points from candidates. An other solution would be to compute a "risk level" for every candidate triangle, and to give triangles with a high risk level a penalty when performing the minimization. The strength of the penalty could be chosen by the radiologist, from 0 to $100 \%$ penalty, the latter leading to a total elimination of the risky insertion points.

Another criterion that could be taken into account is the distance between the chosen entry point and the tumor, as in some cases a radiologist may prefer a more direct insertion. But in some other cases, if the tumor is located close to the capsule, the radiologist would choose a trajectory including a portion of healthy liver tissue instead of a direct access to avoid a possible hemorrhage. Many other criteria like these ones can be cited, and the planning process would benefit if they were included in the process. That is why we plan to work on the integration of these numerous and not always quantifiable constraints.

We also plan to find ways to include those various informations into the interface, in order to help the radiologist if he wants to choose himself among the possible trajectories or even among the proposed insertion zones. The major problem is the amount of necessary information that would be added to the visualization area. To avoid an overload of the visual information, that is very rich yet with the view of the volumic data, we are currently studying the approach of using haptic interfaces for the materialization of extra information.

Finally, we would also like to try to speed up the process. At first, we considered the idea to eliminate very small regions, for instance $<5 \mathrm{~cm}^{2}$, that would sometimes reduce significantly the computation time. But we decided to keep them because for some cases a very optimal solution could be found in one of those very small regions, and according to radiologists the small size of a region is not really a problem to reproduce. Moreover, as we plan to couple our method with a robot 14, trajectories in these zones can easily be reproduced.

\section{Conclusion}

In this paper, we presented an algorithm that automatically computes an optimal needle trajectory, for the planning of a RFA intervention. This algorithm first selects the possible entry zones on the skin, i.e. the zones from which we can reach the tumor without meeting any organ, then computes for each zone the trajectory minimizing the volume of the necrosis zone covering the whole target.

In the future, we plan to improve the algorithm by integrating other criteria in the planning process, as the minimization of the volume is sometimes not the only factor that is taken into account by radiologists to consider a trajectory as optimal, always keeping in mind the reproducibility of the proposed trajectory. 


\section{References}

1. McGahan, J., Dodd III, G.: Radiofrequency ablation of the liver: Current status. American Journal of Roentgenology 176 (2001) 3-16

2. Cady, B., Jenkins, R., Steele Jr, G., et al.: Surgical margin in hepatic resection for colorectal metastasis: a critical and improvable determinant of outcome. Annals of Surgery 227 (1998) 566-571

3. De Baere, T., Denys, A., Wood, B., Lassau, N., Kardache, M., Vilgrain, V., Menu, Y., Roche, A.: Radiofrequency liver ablation: Experimental comparative study of water-cooled versus expandable systems. American Journal of Roentgenology 176 (2001) 187-192

4. Rossi, S., Garbagnati, F., Lencinoni, R.: Unresectable hepatocellular carcinoma: percutaneous radiofrequency thermal ablation after occlusion of tumor blood supply. Radiology 217 (2000) 119-126

5. Poon, R., Ng, K., Lam, C., Ai, V., Yuen, J., Fan, S., Wong, J.: Learning curve for radiofrequency ablation of liver tumors: Prospective analysis of initial 100 patients in a tertiary institution. Annals of Surgery 239 (2004) 441-449

6. Antoch, G., Kuehl, H., Vogt, F., Debatin, J., Stattaus, J.: Value of ct volume imaging for optimal placement of radiofrequency ablation probes in liver lesions. Journal of Vascular and Interventional Radiology 13 (2002) 1155-1161

7. Butz, T., Warfield, S., Tuncali, K., Silverman, S., van Sonnenberg, E., Jolesz, F., Kikinis, R.: Pre- and intra-operative planning and simulation of percutaneous tumor ablation. In: Medical Image Computing and ComputerAssisted Intervention, LNCS 1935. (2000) 317-326

8. Tungjitkusolmun, S., Staelin, S., Haemmerich, D., Cao, H., Tsai, J.Z., Cao, H., Webster, J., Lee, F., Mahvi, D., Vorperian, V.: Three-dimensional finite element analyses for radio-frequency hepatic tumor ablation. IEEE Trans. Biomed. Eng. 49 (2002) 3-9

9. Tombropoulos, R., Adler, J., Latombe, J.: Carabeamer: A treatment planner for a robotic radiosurgical system with general kinematics. Medical Image Analysis 3 (1999) 237-264

10. Soler, L., Delingette, H., Malandain, G., Montagnat, J., Ayache, N., Koehl, C., Dourthe, O., Malassagne, B., Smith, M., Mutter, D., Marescaux, J.: Fully automatic anatomical, pathological, and functional segmentation from ct scans for hepatic surgery. Computer Aided Surgery 6 (2001) 131-142

11. Villard, C., Soler, L., Papier, N., Agnus, V., Thery, S., Gangi, A., Mutter, D., Marescaux, J.: Virtual radiofrequency ablation of liver tumors. In: International Symposium on Surgery Simulation and Soft Tissue Modeling (IS4TM), LNCS 2673. (2003) 366-374

12. Villard, C., Soler, L., Gangi, A., Mutter, D., Marescaux, J.: Towards realistic radiofrequency ablation of hepatic tumors $3 \mathrm{~d}$ simulation and planning. In: Medical Imaging 2004 : Visualization, Image-Guided Procedures, and Display, SPIE 5367. (2004) 586-595

13. Press, W., Teukolsky, S., Vetterling, W., Flannery, B.: Numerical Recipes in C++: The Art of Scientific Computing (Second Edition). (2002)

14. Maurin, B., Gangloff, J., Bayle, B., de Mathelin, M., Piccin, O., Zanne, P., C., D., Soler, L., Gangi, A.: A parallel robotic system with force sensors for percutaneous procedures under ct-guidance. In: Medical Image Computing and ComputerAssisted Intervention MICCAI 2004, LNCS 3217. (2004) 176-183 